\title{
Interacciones potenciales entre productos fitoterápicos con laxantes antracénicos y otros fármacos. Detección en farmacias comunitarias de Sevilla
}

\author{
Fernando Caballero Barbero', María Dolores García Giménez², Ana María Quílez Guerrero² \\ 1. Farmacéutico comunitario. Camas (Sevilla). 2. Profesora Departamento Farmacología, Facultad de Farmacia, Universidad de Sevilla.
}

\section{PALABRAS CLAVE}

Fitoterapia, farmacología, atención farmacéutica, laxantes, hipopotasemia

\section{ABREVIATURAS}

CA: complemento alimenticio CGCOF: Consejo General de Colegios Oficiales de Farmacéuticos EMA: European Medicines Agency ESCOP: European Scientific Cooperative On Phytotherapy FRI: factor de riesgo para la interacción INFITO: Centro de Investigación sobre Fitoterapia

PRM: problema relacionado con el medicamento

RNM: resultado negativo asociado a la medicación

TdP: torsión de puntas

\section{KEYWORDS}

Phytotherapy, pharmacology, pharmaceutical services, laxatives, hypokalemia

\section{RESUMEN}

Introducción: Los productos fitoterápicos más dispensados en España son los laxantes. Los laxantes antracénicos como el acíbar de aloe (Aloe spp.) o la corteza de cáscara sagrada (Rhamnus purshianus), entre otros, podrían estar implicados en interacciones farmacológicas debido al riesgo de hipopotasemia que produce su uso continuado.

Objetivos: Realizar un análisis de la situación de dispensación de estos laxantes y detectar potenciales interacciones con otros fármacos en farmacias de la Provincia de Sevilla.

Material/Métodos: Estudio descriptivo, observacional, realizado desde 14 farmacias comunitarias, mediante aplicación de un cuestionario acerca del uso de plantas medicinales. Se identificaron las interacciones potenciales descritas en las monografías EMA y ESCOP, y se analizaron los factores de riesgo para las mismas (edad avanzada, polimedicación y frecuencia de consumo).

Resultados: La muestra fue constituida por 252 pacientes. El 24,6\% (n=62) consumían laxantes antracénicos, más del 50\% de forma diaria. El porcentaje de asociación con otros fármacos fue del 70\%. Se han identificado potenciales interacciones en el 40\% $(n=26)$ de los pacientes, basadas en la asociación del laxante con fármacos diuréticos, con fármacos que podrían prolongar el intervalo QT, o con ambos a la vez. En todos ellos se detectó la existencia de uno o varios Factores de Riesgo para la Interacción (FRI).

Conclusiones: Los resultados obtenidos reflejan la necesidad de formación actualizada por parte de los farmacéuticos comunitarios sobre los beneficios y riesgos de estos fitomedicamentos. Su dispensación protocolizada favorecería la identificación de posibles interacciones farmacológicas y su uso seguro y racional.

Potential interactions between phytotherapycs anthracenics laxatives products and other drugs. Detection in community pharmacies of Seville

\section{ABSTRACT}

Background: The most dispensed phytotherapeutic products in Spain are laxatives. Anthracenic laxatives such as aloe vera (Aloe spp.) or cascara sagrada (Rhamnus purshianus) could be involved in drug interactions due to the risk of hypokalemia caused by their continued use.

Objectives: To carry out an analysis of the dispensing situation of these laxatives and detect potential interactions in pharmacies of the Province of Seville.

Material / Methods: A descriptive, observational study made from 14 Pharmacy Offices, through the application of a questionnaire about the use of medicinal herbs. The potential interactions described in the EMA and ESCOP monographs were identified, and the risk factors for them (advanced age, polymedication and frequency of use) were analyzed.

Results: The sample was constituted by 252 patients. $24.6 \%(n=62)$ consumed anthracene laxatives, more than 50\% daily. The percentage of association with other drugs was $70 \%$. Potential interactions have been identified in $40 \%(n=26)$ of the patients, based on the association of the laxative with diuretic drugs, with drugs that could prolong the QT interval, or with both at the same time. In all of them, the existence of one or several Risk Factors for Interaction (FRI) was detected.

Conclusions: The results obtained show the need for updated training by community pharmacists on the benefits and risks of these phytoterapics products. Its protocolized dispensation would favor the identification of possible pharmacological interactions and their safe and rational use. 


\section{Introducción}

El uso de la fitoterapia para la prevención y mejora de problemas de salud así como en el tratamiento de patologías crónicas es muy frecuente y sigue una tendencia creciente en nuestra sociedad (1). El potencial de interacción de los preparados fitoterápicos con otros fármacos supone una preocupación para los profesionales de la salud en la actualidad (1).

Factores como la edad, la polimedicación, el empleo de medicamentos de estrecho margen terapéutico, o los polimorfismos genéticos pueden favorecer la aparición de Resultados Negativos a la medicación (RNM) en la administración conjunta de fitoterápicos y otros medicamentos (2).

A menudo, la calidad de los estudios acerca de estas interacciones no es la adecuada, ya que ofrecen algunos resultados contradictorios y no siempre están basados en evidencias clínicas, o bien existe una falta de información en la descripción del producto fitoterápico, por lo que es necesaria una evaluación equilibrada y crítica de las evidencias disponibles para establecer las interacciones que son realmente relevantes (3). Las monografías de la EMA (European Medicines Agency) y de ESCOP (European Scientific Cooperative on Phytotherapy) representan buenos ejemplos de esta visión, siendo las de referencia en la actualidad y considerando únicamente relevantes las interacciones evidenciadas clínicamente en humanos y en estudios de calidad (4).

Los productos fitoterápicos más dispensados en España son los laxantes y adelgazantes (5). Dentro de este grupo, los laxantes estimulantes o hidroxiantracénicos, co mo son el acíbar de aloe (Aloe spp.), las hojas de sen (Cassia spp.), las cortezas de frángula (Rhamnus frangula) y de cáscara sagrada (Rhamnus purshianus) o el rizoma de ruibarbo (Rheum spp.), podrían estar implicados frecuentemente en interacciones con otros fármacos debido al riesgo de hipopotasemia que produce su uso continuado. Como parte de su mecanismo de acción, los glucósidos antraquinónicos contenidos en estas drogas vegetales inhiben la actividad de la ATPasa $\mathrm{Na} / \mathrm{K}$ de los enterocitos, provocando una inhibición de la absorción de agua, sodio y cloro y un aumento de la secreción de potasio a nivel de la mucosa intestinal (6). Esta hipokalemia puede potenciar la acción de heterósidos cardiotónicos como la digoxina, e interaccionar con fármacos antiarritmicos y fármacos que inducen una prolongación del intervalo QT (7). El uso concomitante con medicamentos que provocan eliminación de potasio como diuréticos o corticoides puede ocasionar un desequilibrio electrolítico (8).

El sindrome de QT largo es una alteración de la actividad eléctrica del corazón, de origen hereditario o que puede estar inducido por ciertos medicamentos mediante el bloque de los canales de potasio cardíacos. Esto predispone a la aparición de arritmias ventriculares malignas del tipo de “Torsión de puntas" (TdP) que, en algunos casos, desencadena fibrilación auricular o muerte súbita (9). Entre los medicamentos más conocidos que prolongan el intervalo QT están los antiarrítmicos, pero se ha observado que hay más fármacos que presentan este efecto secundario como algunos antihistamínicos, antibióticos, antivirales, antimicóticos, antieméticos, neurolépticos y antidepresivos, entre otros (10). La incidencia de la TdP producida por medicamentos se presupone que es muy baja, pero podría aumentar por la combinación de fármacos que prolongan el intervalo QT y por factores predisponentes en el paciente, entre los que se incluye el sexo (mayor en las mujeres 2-3:1) y la Hipopotasemia (Potasio sérico <3,5 mg/dl) (11).

En este contexto, los farmacéuticos comunitarios pueden encontrarse con frecuencia en la situación de tener que ofrecer consejo farmacéutico a pacientes demandantes de productos a base de laxantes hidroxiantracénicos que, además, consumen otros medicamentos.

El objetivo de este trabajo es analizar la dispensación de estos productos fitoterápicos en farmacias comunitarias de la Provincia de Sevilla, para poder detectar posibles Problemas relacionados con la administración de medicamentos (PRM) o Resultados Negativos a la medicación (RNM) derivados de su uso concomitante con otros fármacos, y aumentar la seguridad en la Atención Farmacéutica de los pacientes.

\section{Material y métodos}

En primer lugar, se realizó una búsqueda bibliográfica en las mo- nografías EMA $(8,12,13,14,15) \mathrm{y}$ ESCOP $(16,17)$, así como en el Catálogo de Plantas Medicinales del CGCOF (18), acerca de las interacciones descritas entre laxantes hidroxiantracénicos y otros fármacos (tabla 1). Posteriormente, se realizó el estudio a nivel de farmacias comunitarias.

\section{Ámbito de estudio}

Se seleccionaron 14 farmacias del Área Metropolitana de Sevilla. El periodo de estudio fue de mayo de 2016 a abril de 2017, durante el horario habitual de apertura de farmacia.

\section{Diseño}

Se realizó un estudio descriptivo observacional basado en la información aportada por los pacientes consumidores de productos fitoterápicos, a través de un cuestionario acerca del uso de plantas medicinales (figura 1). Las variables fueron:

- Variables sociodemográficas: edad, sexo y nivel de estudios

- Variables clínicas y farmacológicas: Problemas de salud, consumo de plantas medicinales, frecuencia de consumo, nombre de la Planta medicinal o el Producto fitoterápico, a quién pide consejo, lugar de adquisición, motivo del consumo, eficacia del tratamiento, reacciones adversas o alergias, y consumo de otros medicamentos.

Los ítems del cuestionario aplicado, la formulación de preguntas y los formatos de respuestas han sido seleccionados con vistas a los objetivos de detectar PRM o RNM, y han sido validados comprobando una adecuada comprensión y fiabilidad (19).

\section{Muestra}

Los pacientes que solicitaron productos fitoterápicos o los aceptaron en una indicación farmacéutica en la oficina de farmacia. Se excluyeron aquellos con alteraciones cognitivas que dificultasen la comunicación con el entrevistador y los que no quisieron participar en el estudio.

\section{Análisis de datos}

Los datos obtenidos se procesaron en Microsoft Office Excel para 
su posterior análisis descriptivo. Este análisis se mostró utilizando tablas de frecuencia y porcentajes, gráficos de barras y de sectores circulares. Se seleccionaron aquellos pacientes que consumían de manera concomitante un laxante hidroxiantracénico y un medicamento cuya posible interacción farmacológica estuviera recogida en las monografías de EMA o ESCOP (tabla 1), y se analizaron individualmente los Factores de Riesgo para la Interacción (FRI): la edad avanzada, la polimedicación y el consumo diario del laxante.

Tabla 1 Interacciones entre plantas medicinales con laxantes hidroxiantracénicos y medicamentos descritas en las monografías EMA y ESCOP, asi como en el Catálogo de Plantas Medicinales del CGCOF

\begin{tabular}{|c|c|c|c|}
\hline \multicolumn{4}{|c|}{ GRUPO A: APARATO DIGESTIVO } \\
\hline $\begin{array}{l}\text { Fitomedicamento } \\
\text { (Planta, especie, familia } \\
\text { y droga) }\end{array}$ & EMA & ESCOP & Catálogo plantas medicinales \\
\hline $\begin{array}{l}\text { Aloe } \\
\text { Aloe ferox } \\
\text { (Xanthorrhoeaceae) } \\
\text { Droga: Acibar }\end{array}$ & $\begin{array}{l}\text { Produce hipokalemia a lar- } \\
\text { go plazo, lo que potencia la } \\
\text { acción de heterósidos car- } \\
\text { diotónicos e interactúa con } \\
\text { antiarritmicos y fármacos } \\
\text { que inducen la reversión a } \\
\text { ritmo sinusal (quinidina) o } \\
\text { que inducen una prolonga- } \\
\text { ción del intervalo OT. El uso } \\
\text { concomitante con otros me- } \\
\text { dicamentos que provocan } \\
\text { eliminación de potasio (diu- } \\
\text { réticos o corticoides) puede } \\
\text { agravar el desequilibrio elec- } \\
\text { trolítico (EMA-HMPC, 2006). }\end{array}$ & $\begin{array}{l}\text { İdem que reportadas en } \\
\text { EMA (ESCOP, 2009). }\end{array}$ & $\begin{array}{l}\text { - De forma general podría } \\
\text { disminuir la absorción de } \\
\text { cualquier fármaco admi- } \\
\text { nistrado vía oral. } \\
\text { - Digoxina: puede aumen- } \\
\text { tar su toxicidad derivada } \\
\text { de la hipopotasemia. } \\
\text { - Fármacos hipokalemian- } \\
\text { tes: agonistas beta adre- } \\
\text { nérgicos, amfotericina } \\
\text { B, corticoides, diuréticos } \\
\text { tiazídicos o del asa: au- } \\
\text { menta el riesgo de hipopo- } \\
\text { tasemia. } \\
\text { - Prolongadores del in- } \\
\text { tervalo 0T: antagonista } \\
\text { 5-HT3, antidepresivos, } \\
\text { antihistamínicos H1, for- } \\
\text { moterol, ivabradina, ma- } \\
\text { crólidos, neurolépticos: } \\
\text { junto con la hipopotase- } \\
\text { mia, aumenta el riesgo de } \\
\text { arritmias cardíacas. }\end{array}$ \\
\hline $\begin{array}{l}\text { Cáscara sagrada } \\
\text { Rhamnus purshianus } \\
\text { (Rhamnaceae) } \\
\text { Droga: Corteza }\end{array}$ & $\begin{array}{l}\text { İdem que Aloe } \\
\text { (EMA-HMPC, 2007) }\end{array}$ & $\begin{array}{l}\text { İdem que Aloe } \\
\text { (ESCOP, 2003) }\end{array}$ & İdem que Aloe. \\
\hline $\begin{array}{l}\text { Frángula } \\
\text { Rhamnus frangula } \\
\text { (Rhamnaceae) } \\
\text { Droga: Corteza }\end{array}$ & $\begin{array}{l}\text { İdem que Aloe } \\
\text { (EMA-HMPC, 2006) }\end{array}$ & $\begin{array}{l}\text { İdem que Aloe } \\
\text { (ESCOP, 2003) }\end{array}$ & İdem que Aloe. \\
\hline $\begin{array}{l}\text { Ruibarbo } \\
\text { Rheum palmatum } \\
\text { (Polygonaceae) } \\
\text { Droga: Raíz }\end{array}$ & $\begin{array}{l}\text { İdem que Aloe } \\
\text { (EMA-HMPC, 2007). }\end{array}$ & $\begin{array}{l}\text { İdem que Aloe } \\
\text { (ESCOP, 2003). }\end{array}$ & İdem que Aloe. \\
\hline $\begin{array}{l}\text { Sen } \\
\text { Cassia angustifolia } \\
\text { (Leguminosae) } \\
\text { Droga: hoja y fruto }\end{array}$ & $\begin{array}{l}\text { İdem que Aloe } \\
\text { (EMA-HMPC, 2006). }\end{array}$ & $\begin{array}{l}\text { İdem que Aloe } \\
\text { (ESCOP, 2003). }\end{array}$ & Ídem que Aloe. \\
\hline
\end{tabular}




\section{USO DE PLANTAS MEDICINALES EN FARMACIA}

\begin{tabular}{|l|l|l|l|}
\hline EDAD: $\square<20 \square 20-50 \quad \square$ 50-70 $\square>70$ \\
\hline SEXO: $\square$ HOMBRE $\square$ MUJER & \\
\hline NIVEL DE ESTUDIOS: $\square$ BÁSICOS $\square$ SUPERIORES $\square$ INTERMEDIOS \\
\hline
\end{tabular}

$>$ ¿PADECE USTED ALGUN PROBLEMA DE SALUD?

$\square$ HIPERTENSIÓN

ARTERIAL

$\square$ DIABETES

$\square$ COLESTEROL ALTO

$\square$ OBESIDAD $\square$ ENFERMEDAD RESPIRATORIA

$\square$ PROBLEMAS DE TIROIDES

$\square$ DEPRESIÓN

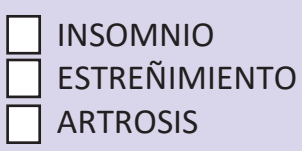

ARTROSIS

OTRAS:

$>$ ¿CONSUME O HA CONSUMIDO ALGUNA VEZ PLANTAS MEDICINALES? ¿CON QUÉ FRECUENCIA? $\square$ DIARIAMENTE $\square$ SEMANALMENTE $\square$ OCASIONALMENTE PLANTA MEDICINAL O NOMBRE DEL PREPARADO:

$>$ ¿A QUIÉN PIDE CONSEJO ACERCA DEL USO DE LAS PLANTAS MEDICINALES?

$\square$ A NADIE, LAS TOMA POR SU CUENTA

$\square$ PERSONAL DE HERBORISTERIA / SUPERMERCADO

$\square$ MÉDICO

FARMACÉUTICO

$\square$ OTROS (INTERNET, AMIGOS..)

$>$ ¿DÓNDE ADQUIERE NORMALMENTE LAS PLANTAS MEDICINALES QUE CONSUME?

$\square$ FARMACIA

PARAFARMACIA

HERBORISTERIAS

$\square$ SUPERMERCADOS
$\square$ INTERNET

$>$ ¿POR QUÉ EMPEZÓ A USAR PLANTAS MEDICINALES?

$\square$ PARA TRATAR ALGÚN PROBLEMA DE SALUD MENOR

$\square$ POR FALTA DE EFECTIVIDAD DEL TRATAMIENTO PRESCRITO POR EL MÉDICO

$\square$ PARA TRATAR UN EFECTO ADVERSO DEL TRATAMIENTO PRESCRITO POR EL MÉDICO

$\square$ OTRAS CAUSAS:

$>\quad$ ¿LE ES EFICAZ EL TRATAMIENTO A BASE DE PLANTAS MEDICINALES? $\square \mathrm{SI} \square$ NO $\square$ REGULAR

$>$ ¿HA NOTADO ALGUNA VEZ UN EFECTO ADVERSO O ALERGIA AL CONSUMIR PLANTAS MEDICINALES? ¿CUÁL/ES? $\square$ SI $\square$ NO

$>$ ¿CONSUME OTROS MEDICAMENTOS ADEMÁS DE LAS PLANTAS MEDICINALES? ¿CUÁL/ES? $\square \mathrm{SI} \quad \square$ NO

\section{¡iMUCHAS GRACIAS POR SU ATENCIÓN!!}

Figura 1 Cuestionario acerca del uso de plantas medicinales empleado para el estudio a nivel de farmacia 


\section{Resultados}

- Se procesaron un total de 252 cuestionarios de un total de 270 cumplimentados, excluyéndose los incompletos. En 14 de los 18 cuestionarios excluidos las preguntas no contestadas fueron las relativas al nombre de la planta medicinal o producto fitoterápico consumido y a la asociación con otros medicamentos.

- El 24,6\% de los pacientes encuestados $(n=62)$ consumía algún producto fitoterápico a base de laxantes hidroxiantracénicos, entre los que destacan los preparados a base de acíbar de aloe $(62,9 \%$ de los 62 pacientes), hojas y frutos de sen $(24,2 \%)$ o corteza de cáscara sagrada $(11,3 \%)$. Del análisis de datos de este grupo de pacientes se obtuvo la siguiente información:

- Los mayores consumidores de estos laxantes son las mujeres de 20-50 años y de 50-70 años de edad (tabla 2).

- El 75\% de los pacientes que consumían los laxantes lo hacían por recomendación de un profesional sanitario (el 58\% por parte de un farmacéutico y el 14,5\% por prescripción médica). La cuarta parte restante utilizaba canales alternativos como herboristerías $(9,75 \%)$ o directamente automedicándose (11,75\%).

- En la distribución del tipo de registro sanitario de los fitoterápicos laxantes consumidos, se observa una mayoría de complementos alimenticios (CA) con un 74\%, frente a un $26 \%$ de medicamentos.

- El 58\% de los pacientes tomaban estos productos diariamente; el resto, con una frecuencia semanal

Tabla 2 Distribución de los pacientes consumidores de laxantes hidroxiantracénicos por sexo y rango de edad.

\begin{tabular}{|l|c|c|}
\hline Rango de edad (años) & Hombres & Mujeres \\
\hline Menores (<20 años) & 0 & 0 \\
\hline Adultos jóvenes (20-50 años) & 1 & 24 \\
\hline Adultos maduros (50-70 años) & 1 & 20 \\
\hline Mayores (>70 años) & 6 & 10 \\
\hline Total & $8(13 \%)$ & $54(87 \%)$ \\
\hline
\end{tabular}

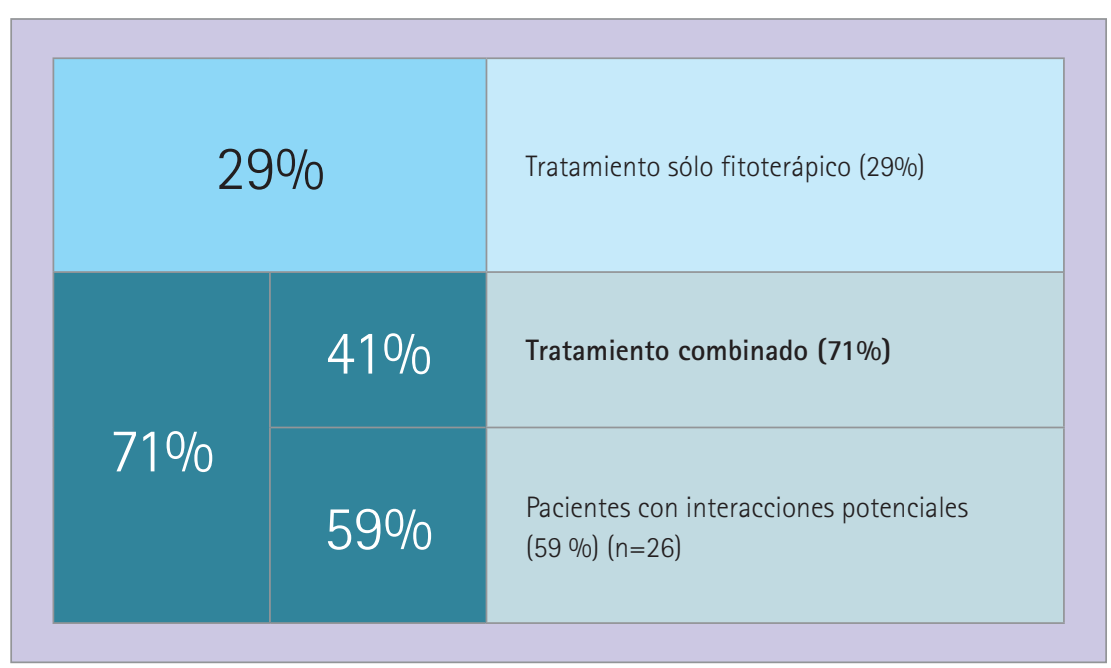

Figura 2 Distribución de los tratamientos fitoterápicos a base de laxantes antracénicos con o sin medicamento/s asociados

(16\%) u ocasional (26\%). Sólo 3 pacientes manifestaron sufrir algún efecto adverso, que en estos casos fue diarrea.

- En cuanto al motivo de consumo, el $72,6 \%$ los tomaba para tratar un problema de salud menor o el síntoma de otra enfermedad, que en este caso era el estreñimiento; y más del 80\% consideraba que el tratamiento a base de estos laxantes le era eficaz.
- El 71\% (n=44) consumían de forma concomitante otros fármacos de prescripción, identificándose interacciones potenciales en el $42 \%(n=26)$ de los pacientes que tomaban estos laxantes (figura 2). Entre estos pacientes se detectaron factores que podrían aumentar el riesgo de interacción (figura 3). En todos ellos se detectó al menos la existencia de un Factor de Riesgo para la Interacción (FRI), y en

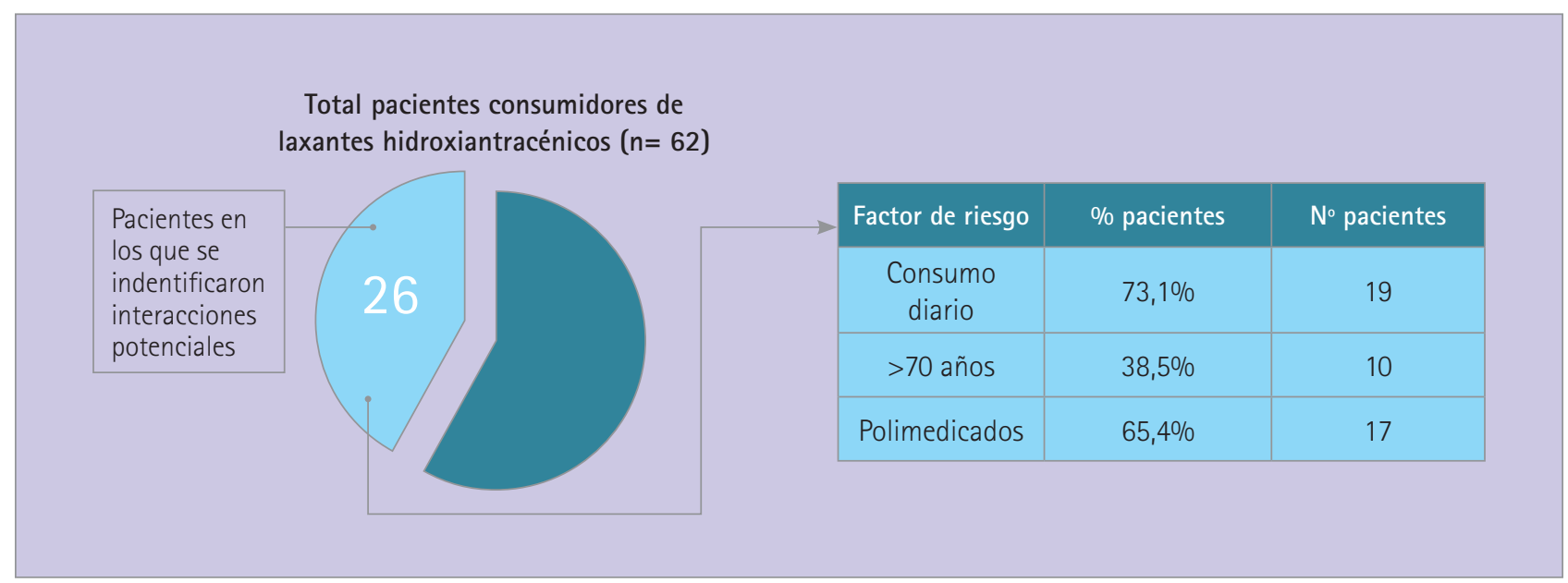

Figura 3. Proporción de pacientes en los que se identificaron interacciones potenciales y factores de riesgo. 


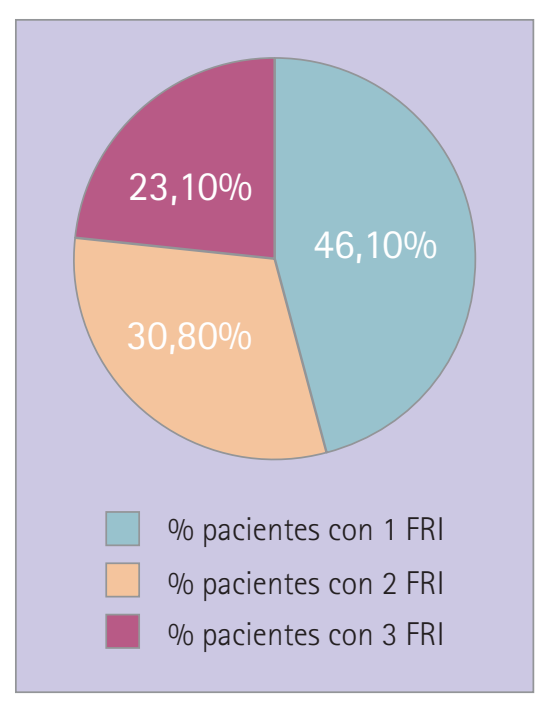

Figura 4 Prevalencia de Factores de Riesgo para la Interacción (FRI) entre los pacientes en los que se identificaron interacciones potenciales entre el fitoterápico laxante antracénico y otro/s fármaco/s

algunos casos concurrían 2 o 3 FRI (figura 4).

- Las interacciones potenciales se basaban en la asociación del laxante hidroxiantracénico con fármacos diuréticos $(n=13)$, con fármacos que podrían prolongar el intervalo QT $(n=9)$, o con ambos a la vez $(n=3)$. Sólo en un paciente se identificó una sinergia, al tener en su tratamiento un fármaco de síntesis indicado también para el estreñimiento (tabla 3).

\section{Discusión}

Los resultados del estudio no se pueden extrapolar a la totalidad de la población de Sevilla, ya que sólo podemos describir los resultados de una muestra pequeña de pacientes. No obstante, el objetivo perseguido fue realizar un análisis de la situación de dispensación de los laxantes antracénicos en algunas farmacias comunitarias y detectar posibles interacciones farmacológicas, y no un análisis poblacional como tal.

Comparando los resultados de nuestra encuesta con otros trabajos previos, los datos sobre el consumo y el perfil del paciente coinciden con los obtenidos en otros estudios de dispensación de productos fitoterápicos realizados en España (20-21), en los que se observa que los productos fitoterápicos más empleados son los indicados en trastornos digestivos, en concreto aquellos con propiedades laxantes y adelgazantes, y que 3 de cada 4 consumidores son mujeres (22).

Un gran porcentaje de pacientes (58\%) consume estos laxantes por recomendación del farmacéutico, pero hay que considerar que el estudio se ha realizado desde farmacias comunitarias, lugar donde habitualmente compran estos productos, por lo que cabe destacar que más del 20\% utilicen otros canales alternativos como herboristerías o Internet. De hecho, el estudio INFITO reveló que el herbolario es el lugar que concita un mayor número de compradores de fitoterapia (22). Aún así, estos datos reflejan la gran labor asistencial del farmacéutico en cuanto al uso racional de plantas medicinales, y la importancia que debe tener el conocimiento de los posibles riesgos e interacciones farmacológicas que puedan darse derivadas de su consumo.

En cuanto a la frecuencia de consumo, más de la mitad de los pacientes toman estos laxantes de forma diaria, dato que cobra especial importancia en nuestro análisis, ya que es el uso continuado de los derivados antracénicos lo que aumenta el riesgo de hipopotasemia y las consiguientes interacciones farmacológicas. En este sentido, se debe hacer mayor hincapié desde las farmacias en que estos productos fitoterápicos son seguros y eficaces cuando se emplean durante cortos periodos de tiempo (evitando tratamientos superiores a una semana), pero que su utilización sin el debido control sanitario puede originar dependencia, atonía intestinal o, por el contrario, la llamada "enfermedad de los laxantes" (diarreas, náuseas, dolores abdominales, etc.) (18). En nuestro estudio, el número de pacientes que ha experimentado algún RNM tras la ingesta de laxantes antracénicos fue bajo, tan solo 3 y con sintomas de diarrea, pero pone de manifiesto que los riesgos del abuso de estos productos existen.

El motivo de consumo en el $72,6 \%$ de los pacientes fue tratar un problema de salud menor, que presumiblemente sería el estreñimiento, pero se observa que en muchos casos los productos demandados hacen referencia a propiedades adelgazantes y antiobesidad. Estos son productos fitoterápicos registrados como Com- plementos Alimenticios (CA), cuya venta es libre y no son competencia de la Agencia Española del Medicamento, y que en nuestro estudio fueron los que mayoritariamente usaban los participantes encuestados frente a los productos registrados como Medicamentos. Esto podría traducirse en que hay pacientes que consumen estos fitoterápicos de forma indebida e insegura. Su empleo en tratamientos para el sobrepeso está desaconsejado puesto que a los CA no se les puede atribuir propiedades curativas ni de prevención, ya que no son medicamentos. El consejo farmacéutico debe ser emplearlos siempre de forma ocasional, limitando su uso a casos puntuales de estreñimiento o cuando se requiere una evacuación del intestino con heces blandas (postoperatorios, hemorroides etc.) (23).

La percepción de eficacia del tratamiento fitoterápico en nuestro estudio fue bastante buena. Más del 80\% de los pacientes consideró que su tratamiento a base de laxantes le era eficaz, datos concordantes con los obtenidos en estudios nacionales, en los que el 96,2\% de los pacientes que compraban las plantas medicinales en la farmacia se mostró muy o bastante satisfecho con su tratamiento (22).

El porcentaje de pacientes con tratamiento fitoterápico asociado a otros medicamentos, un 71\%, fue bastante elevado si lo comparamos con un estudio realizado en la provincia de Sevilla en 2011, en el que se obtuvo tan solo un $15,4 \%$ de asociación (24). A pesar de que prácticamente no existan casos descritos de interacciones farmacológicas derivadas del abuso de laxantes antracénicos, teóricamente es probable que ocurran (25). En el presente estudio no se han podido identificar casos de interacciones farmacológicas reales; tan solo se han analizado los factores de riesgo y las interacciones teóricas que podrian darse en los diferentes pacientes. De esta forma, se han identificado riesgos de interacción entre el laxante y el/los fármaco/s en 26 pacientes, esto es en el 42\% del total de consumidores de estos productos fitoterápicos, un número que puede considerarse elevado teniendo en cuenta el pequeño tamaño de la muestra. Si partimos de la base de que es el uso continuado de estos laxantes lo que produce las posibles interacciones, también encontramos 
Tabla 3. Información obtenida a través de los cuestionarios sobre los pacientes en los que se identificaron interacciones potenciales entre laxantes hidroxiantracénicos y otros medicamentos.

\begin{tabular}{|c|c|c|c|c|c|c|c|c|c|}
\hline & $\begin{array}{l}\text { Franja } \\
\text { etaria }\end{array}$ & Sexo & F.C. & Reg. & $\begin{array}{c}\text { Planta } \\
\text { medicinal }\end{array}$ & Consejo & $\begin{array}{c}\text { Fármaco/s } \\
\text { con interacción } \\
\text { potencial }\end{array}$ & $\begin{array}{l}\text { Descripción } \\
\text { interacción }\end{array}$ & N \\
\hline 1 & $50-70$ & M & D & CA & Aloe vera & Farmacéutico & $\operatorname{Tmp} / \mathrm{Smx}$ & Prolonga qt & 1 \\
\hline 2 & $>70$ & M & D & CA & Aloe vera & Farmacéutico & Furosemida (PMed) & Hipokalemia & 3 \\
\hline 3 & $50-70$ & M & D & CA & Aloe vera & Farmacéutico & $\begin{array}{l}\text { Hctz, Citalopram } \\
\text { (PMed) }\end{array}$ & $\begin{array}{l}\text { Prolonga qt + } \\
\text { Hipokalemia }\end{array}$ & 2 \\
\hline 4 & $>70$ & $\mathrm{H}$ & 0 & MTP & Aloe vera & Nadie & Lactulosa (PMed) & Sinergia + & 2 \\
\hline 5 & $50-70$ & M & D & CA & Aloe vera & Farmacéutico & Hctz (PMed) & Hipokalemia & 2 \\
\hline 6 & $50-70$ & $M$ & D & CA & Aloe vera & Farmacéutico & Hctz (PMed) & Hipokalemia & 2 \\
\hline 7 & $>70$ & $H$ & D & CA & Aloe vera & Otros & Hctz (PMed) & Hipokalemia & 3 \\
\hline 8 & $>70$ & M & 0 & CA & Aloe vera & Medico & Hetz & Hipokalemia & 1 \\
\hline 9 & $>70$ & M & D & MTP & Aloe vera & Farmacéutico & Clortalidona (PMed) & Hipokalemia & 3 \\
\hline 10 & $50-70$ & M & 0 & MTP & Aloe vera & Farmacéutico & Furosemida (PMed) & Hipokalemia & 1 \\
\hline 11 & $50-70$ & M & 0 & CA & Aloe vera & Farmacéutico & Risperidona (PMed) & Prolonga qt & 1 \\
\hline 12 & $>70$ & M & D & MTP & Aloe vera & Médico & $\begin{array}{l}\text { Clortalidona, } \\
\text { Trazodona (PMed) }\end{array}$ & $\begin{array}{l}\text { Prolonga qt + } \\
\text { Hipokalemia }\end{array}$ & 3 \\
\hline 13 & $>70$ & $\mathrm{H}$ & S & CA & Aloe vera & Farmacéutico & Tizanidina (PMed) & Prolonga qt & 2 \\
\hline 14 & $50-70$ & M & D & CA & Aloe vera & Farmacéutico & Hctz & Hipokalemia & 1 \\
\hline 15 & $>70$ & M & D & CA & Aloe vera & Farmacéutico & Amiodarona (PMed) & Prolonga qt & 3 \\
\hline 16 & $50-70$ & M & 0 & MTP & Cáscara sagrada & Nadie & Hctz (PMed) & Hipokalemia & 1 \\
\hline 17 & $20-50$ & M & D & MTP & Cáscara sagrada & Nadie & Venlafaxina & Prolonga qt & 1 \\
\hline 18 & $20-50$ & M & D & CA & Cáscara sagrada & Farmacéutico & Hetz & Hipokalemia & 1 \\
\hline 19 & $50-70$ & M & D & MTP & Cáscara sagrada & Medico & Furosemida & Hipokalemia & 1 \\
\hline 20 & $50-70$ & M & D & CA & Frángula & Nadie & Escitalopram & Prolonga qt & 1 \\
\hline 21 & $20-50$ & M & D & CA & Sen & Farmacéutico & Furosemida & Hipokalemia & 1 \\
\hline 22 & $50-70$ & M & D & CA & Sen & Farmacéutico & Amitriptilina (PMed) & Prolonga qt & 2 \\
\hline 23 & $>70$ & $\mathrm{H}$ & D & MTP & Sen & Nadie & $\begin{array}{l}\text { Trazodona, Hctz } \\
\text { (PMed) }\end{array}$ & $\begin{array}{l}\text { Prolonga qt + } \\
\text { Hipokalemia }\end{array}$ & 3 \\
\hline 24 & $50-70$ & M & D & CA & Sen & Farmacéutico & Hctz & Hipokalemia & 1 \\
\hline 25 & $>70$ & M & S & MTP & Sen & Nadie & Tolterodina (PMed) & Prolonga qt & 2 \\
\hline 26 & $20-50$ & M & D & MTP & Sen & Médico & $\begin{array}{l}\text { Fluoxetina, } \\
\text { Amitriptilina (PMed) }\end{array}$ & Prolonga qt & 2 \\
\hline
\end{tabular}

Se muestra de izquierda a derecha: Franja etaria, Sexo, Frecuencia de consumo del laxante (F.C.), Tipo de registro sanitario del producto fitoterápico (Reg.) Nombre de la planta medicinal consumida, Fuente de información/consejo acerca de su uso, Fármaco/s con el/los que se produce la interacción potencial, Descripción de la posible interacción y No de factores de riesgo que concurren en el paciente para favorecer la aparición de interacciones (N). Abreviaturas: $\mathrm{H}$ (Hombre), M (Mujer), D (Diaria), S (Semanalmente), O (Ocasionalmente), MTP (Medicamento Tradicional a base de Plantas), CA (Complemento Alimenticio), TMP/ SMX (Trimetoprim/Sulfometoxazol). HCTZ (Hidroclorotiazida). PMed (Polimedicados). 
un alto número de pacientes con este factor de riesgo, ya que 19 de los 26 pacientes mencionados los consumían diariamente.

Analizando las potenciales interacciones detectadas, el $50 \%$ se basan en el riesgo de hipopotasemia y desequilibrio electrolítico que podría presentarse al administrar de forma conjunta el fitoterápico antracénico y un fármaco diurético. Los diuréticos son en muchos casos el tratamiento de primera línea para una de las patologías más frecuentes, la Hipertensión arterial, y su administración suele ser diaria. Por tanto, es coherente que este tipo de interacciones sean las que más frecuentemente podamos detectar desde las oficinas de farmacia.

Respecto a las posibles interacciones con fármacos que prolongan el intervalo QT, se debe considerar que estos se agrupan en tres grupos según el nivel de evidencia disponible de prolongación del intervalo QT y del riesgo de producir TdP (10). Así, de fármacos antiarrítmicos como amiodarona y flecainida, o antidepresivos como citalopram $\mathrm{y}$ escitalopram, las evidencias disponibles apoyan la conclusión de que aumentan los intervalos QT y tienen un riesgo de TdP cuando se utilizan en las indicaciones autorizadas. Otros principios activos como risperidona o venlafaxina pueden causar prolongación del intervalo QT, pero no hay pruebas suficientes de que tengan riesgo de causar TdP cuando se utilizan en las indicaciones autorizadas. En el caso de fármacos como trazodona, fluoxetina o tolterodina, los estudios disponibles concluyen que aumentan el intervalo QT y tienen un riesgo de desarrollar TdP, pero sólo cuando se dan concurrencia de factores de riesgo. En nuestro estudio se han detectado pacientes en los que concurren estos factores de riesgo, como el sexo (el riesgo de prolongación de QT es mayor en las mujeres en una proporción 2-3:1), la polimedicación (aumenta el riesgo de que se produzcan más interacciones o de inhibición del metabolismo enzimático del citocromo P450), la edad (la función hepática y renal puede estar disminuida) o una posible hipopotasemia por administración prolongada de laxantes antracénicos y fármacos diuréticos.

\section{Conclusiones}

Debido al uso extendido de productos fitoterápicos a base de laxantes antracénicos, y en vista de los resultados obtenidos en nuestra muestra poblacional, consideramos importante que los farmacéuticos comunitarios posean información actualizada sobre los beneficios y riesgos de estos fitomedicamentos, identificando las posibles interacciones farmacológicas con otros fármacos de sintesis y promoviendo su uso seguro y racional. Para ello, se hace necesario la realización de un seguimiento farmacoterápico y un protocolo de dispensación e indicación, considerando los factores que favorecen la aparición de RNM con los tratamientos asociados.

\section{Agradecimientos}

Agradecemos a la Vocalía de Plantas medicinales y Homeopatía del Real e Ilustre Colegio Oficial de Farmacéuticos de Sevilla por su apoyo prestado y a los farmacéuticos comunitarios que desinteresadamente han contribuido a este estudio.

\section{Referencias bibliográficas}

1. Vanaclocha B, Risco E, Cañigueral S. Interacciones entre preparados vegetales y fármacos de síntesis: revisión de las monografías EMA y ESCOP. Rev. de Fitoterapia.2014; 14(1):5-36

2. Quílez A, García MD, Sáenz MT. Uso racional de medicamentos a base de plantas. Guía de interacciones entre fitomedicamentos y fármacos de síntesis. $1^{\text {a }}$ ed. Sevilla: Fundación Farmacéutica Avenzoar; 2009

3. Posadzki P, Watson L, Ernst E. Herbdrug interactions: an overwiew of sistematic reviews. Br J Clin Pharmacol. 2012; 75(3): 603-618. https://doi.or$\mathrm{g} / 10.1111 / \mathrm{j} .1365-2125.2012 .04350 . \mathrm{x}$

4. Anon. Interactions with herbal products. Drug Ther Bull.2014;52(2):18-21. https://doi.org/10.1136/dtb.2014.2.0234

5. Portalfarma [www.portalfarma.com]. Madrid: Consejo General de Colegios Oficiales de Farmacéuticos; 2016 [acceso 12 de Julio de 2018]. El mercado de las plantas medicinales en España. Disponible en: http://www.portalfarma.com/Profesionales/campanaspf/ categorias/Paginas/introduccionalafitoterapia.aspx

6. Castillo E, Martínez I. Manual de Fitoterapia. 2a ed. Madrid: Elsevier; 2015.

7. Celaya MC, Martínez J. Medicamentos y prolongación del intervalo QT. Boletín de información farmacoterapéutica de Navarra. 2013; 21(1): 4-6.
8. EMA- HMPC. European Union herbal monograph on Aloe barbadensis Mill. and on Aloe (various species, mainly Aloe ferox Mill. and its hybrids), folii succus siccatus. [Internet]. Londres: 2016 [acceso 4 de Julio de 2018]. Disponible en: http:// www.ema.europa.eu/docs/en_GB/ document_library/Herbal__Herbal_ monograph/2017/04/WC500225529. pdf

9. Medeiros-Domingo A, Iturralde-Torres P, Ackermanc M. Clínica y genética en el síndrome de QT largo. Rev Esp Cardiol. 2007; 60 (7): 739-52. https://doi.org/10.1157/13108280

10. Woosley RL. Drugs that prolong the QT intervalo and/or Induce Torsade de Pointes. [Internet]. Arizona: 2018 [acceso el 5 de Octubre de 2018]. Disponible en www.azcert.org

11. Kallergis EM, Goudis CA, Simantirakis EN, Kochiadakis GE, Vardas PE. Mechanisms, risk factors, and rrhythmia of acquired Long QT Syndrome: a comprehensive review. The Scientific World Journal. 2012; 1: 1-8. https://doi. org/10.1100/2012/212178

12. EMA-HMPC. Community herbal monograph on Rhamnus purshianus DC cortex. London: 2007. Doc. Ref.: EMEA/HMPC/513579/2006.

13. EMA-HMPC. Community herbal monograph on Rhamnus frangula L., cortex. London: 2006 [en línea] [Consultado en Octubre 2018] Disponible en: https://www.ema.europa.eu/documents/ herbal-monograph/final-community-herbal-monograph-rhamnus-frangula-l-cortex_en.pdf

14. EMA-HMPC. Community herbal monograph on Rheum palmatum L., radix. London: 2007 [en línea] [Consultado en Octubre 2018] Disponible en: https://www.ema.europa. eu/documents/herbal-monograph/ final-community-herbal-monograph-rheum-palmatum-l-rheum-officinale-baillon-radix_en.pdf

15. EMA- HMPC. Community herbal monograph on Cassia senna L., Fructus and Cassia angustifolia Vahl, folium. London: 2006 [en línea]. [Consultado en Octubre 2018]. Disponible en: https://www.ema.europa. eu/documents/herbal-monograph/ final-community-herbal-monograph-cassia-senna-l-cassia-angustifolia-vahl-folium_en.pdf

16. ESCOP (European Scientific Cooperative on Phytotherapy). ESCOP Monographs. The Scientific foundation for herbal medicinal products. $2^{\text {a }}$ ed. Exeter (Reino Unido): ESCOP, Stuttgart: Georg Thieme Verlag, y New York: Thieme New York. 2003

17. ESCOP (European Scientific Cooperative on Phytotherapy). ESCOP 
Monographs. The Scientific foundation for herbal medicinal products. $2^{\text {a }}$ ed. Exeter (Reino Unido): ESCOP, Stuttgart: Georg Thieme Verlag, New York: Thieme New York. 2009.

18. Consejo General de Colegios Oficiales de Farmacéuticos. Catálogo de Plantas Medicinales. Colección Consejo 2010. Madrid: CGCOF; 2010.

19. Guix J. Dimensionando los hechos: la encuesta (II). Rev. Calidad Asistencial 2005; 20 (3):154-60. https:// doi.org/10.1016/S1134-282X(08) 747 41-9
20. Hernando B. (Ed). Libro blanco de la herboristería y las plantas medicinales. Soria: Fundación Salud y Naturaleza. 2007

21. Consejo General de Colegios Oficiales de Farmacéuticos. Trastornos digestivos y procesos nerviosos, usos más comunes de las plantas medicinales. Rev Farmacéuticos. 2016; 1 (420): 19.

22. Centro de Investigación sobre fitoterapia [INFITO]. Investigación sobre la tendencia al uso de plantas medicinales con fines terapéuticos. 2011.
23. Centro de Investigación sobre fitoterapia [INFITO]. Plantas medicinales para el sobrepeso. $1^{\text {a }}$ ed. Madrid: Editorial Complutense; 2009.

24. Quílez AM, Domínguez MD, Cuenca F, Justo I, Martin-Calero MJ. Dispensación de preparados fitoterápicos en farmacias comunitarias de Sevilla. Revista de Fitoterapia. 2011; 11(1):53-59

25. Williamson E, Driver S, Baxter K. Stockley's Herbal Medicines Interactions: a guide to the interactions of herbal medicines. $2^{\text {a }}$ ed. Londres: Pharmaceutical Press; 2013. 